Mirai. Estudios Japoneses

ISSN-e: 2531-145X

http://dx.doi.org/10.5209/mira.67537

\title{
Breve recorrido por el emprendimiento social en Japón: Del Japón pre-moderno hasta el Tōhoku post Fukushima
}

\author{
Mario Malo Sanz ${ }^{1}$
}

Resumen. El presente artículo, tiene como objetivo dotar de dimensión analítica al sujeto social conocido como social entrepeneur, desde el Japón pre-moderno hasta sus nuevas formas de desarrollo en el Tōhoku post Fukushima.

Hablamos de una figura, cuya conformación histórica podemos observar de forma intermitente en el Japón preindustrial, llegando hasta la modernidad del era Meiji, y cuya normatividad estructural cambiará sustancialmente tras la primera guerra mundial, para llegar hasta nuestros días bajo diferentes iniciativas. La transformación de las dinámicas coyunturales en el plano socioeconómico y político, unidas al fenómeno de la globalización hacen que el concepto que hoy usamos para definir este tipo de propuesta de desarrollo, esté estrechamente ligado a los procesos que se dan en Europa y Estados Unidos a finales de los años 80, y que empiece a tener cierta transcendencia durante la primera década del XXI, alcanzando cuotas importantes de significación social tras el triple desastre de Fukushima en 2011.

Palabras clave: Tōhoku; emprendimiento social; Japón; sociedad civil; triple desastre de Fukushima.

\begin{abstract}
The purpose of this article is to provide an analytical dimension to the social subject known as social entrepreneur, from pre-modern Japan to its new forms of development in the Tōhoku post Fukushima. We are talking about a figure, whose historical conformation we can observe intermittently in the pre-industrial Japan, reaching the modernity of the Meiji period, and whose structural regulations will change substantially after the First World War, to reach our days under different initiatives. The transformation of contextual dynamics at the socioeconomic and political level, together with the phenomenon of globalization, make the concept we use today to define this type of development proposal closely linked to the processes that occurred in Europe and the United States at the end of the $80 \mathrm{~s}$, and that it began to have some significance during the first decade of the XXI, reaching important quotas of social significance after the triple disaster of Fukushima in 2011.
\end{abstract}

Keywords: Tōhoku; social entrepreneurship; Japan; civil society; triple Fukushima disaster.

Sumario. 1. Marco teórico; 2. Introducción; 3. Trayectoria histórica del emprendimiento social en Japón; 4. Segunda generación de emprendimiento social. 5. Tercera generación y Tōhoku post Fukushima; 6. Tipologías migratorias y población autóctona; 7. Proyectos y orientación de las iniciativas; 8. Conclusión; 9. Bibliografía.

Cómo citar: Malo Sanz, M. Breve recorrido por el emprendimiento social en Japón: Del Japón pre-moderno hasta el Tōhoku post Fukushima, en Mirai. Estudios Japoneses, 4, 2020, 75-85.

\section{Marco teórico}

Utilizaremos un marco teórico de doble prisma sustentado en: el modelo triangular de los movimientos sociales de McAdam (1996) y un "modelo generacional" de emprendimiento social con cierta influencia de la obra de Kogane $(2013)^{2}$. Todo ello, servirá de basamento para encuadrar el trabajo etnográfico de carácter cualitativo, llevado a cabo durante 2017-2018 en el área de las prefecturas de Iwate (Tōno, Rikuzentakata y Kamaishi) y Miyagi (Yamamoto), e intentaremos dar respuesta a las siguientes preguntas: ¿Cuál ha sido la evolución histórica de este tipo de iniciativas en Japón y qué entendemos actualmente por emprendedor social o shakai ikigyōka (社会い起業 家)? ¿Cómo se ha desarrollado el emprendimiento social en la región de Tōhoku post-Fukushima? ¿Cuáles son los principales impulsores en el proceso de desarrollo? ¿Cuáles son las características del aspecto de innovación del emprendimiento social japonés y por qué la innovación es particularmente importante en el contexto demográfico del Tōhoku actual?

\footnotetext{
Doctorando de la Universidad Autónoma de Barcelona.

mariomalo87@hotmail.com

2 McAdam, D. (1996); Kogane, Y. (2013).
} 


\section{Introducción}

Grosso modo, podríamos decir, que, en el mundo económicamente desarrollado, si excluimos la unidad familiar, la familia extensa, y el Estado, tradicionalmente han existido dos tipos de organizaciones encargadas de satisfacer, desde una perspectiva maslowiana, una parte de las necesidades sociales. Por un lado, el ámbito empresarial (grandes empresas en diferentes variantes, corporaciones, PYMES, etc.), y por otro las organizaciones filantrópicas como ONG, $\mathrm{OSAL}^{3}$ y diferentes grupos de la sociedad civil. Tradicionalmente las primeras han basado sus objetivos primordiales en la maximización del beneficio económico a través de la producción de utilidades, mientras que por otro lado las organizaciones sin ánimo de lucro, que a pesar de que hoy en día también ejercen funciones de cuasi mercado sobre todo en Japón y Estados Unidos, dan soluciones a diferentes problemáticas sociales. Ambos modelos tienen lagunas importantes, por un lado, en el mundo empresarial, sí que hay ciertos estatutos sobre responsabilidad social corporativa, cuyo cumplimiento, en general, suele quedar supeditado a las dinámicas del capital y por ende en segundo plano. Cuando se lleva a cabo, en general, muy pocas veces se hace desde una perspectiva altruista, más bien subyacen objetivos utilitaristas relacionados con la proyección de una determinada imagen empresarial. Por otro lado, tenemos las organizaciones filantrópicas o sin ánimo de lucro, que generalmente tienen un problema de escalabilidad, es decir, como se sustentan económicamente en donaciones, en partidas económicas que proceden del Estado o en el capital de sus propios socios, la viabilidad de proyectos a largo plazo, normalmente, suele ser limitada. Debido a estas cuestiones, y fruto de la experiencia participativa en los dos modelos que he mencionado anteriormente surge el sujeto que conocemos como social entrepeneur o emprendedor social.

Cuando intentamos definir lo que es el emprendimiento social, surge cierta confusión entre este y las ONG o incluso con las empresas y sus estatutos de responsabilidad social corporativa. Por un lado, podríamos decir que una de las diferencias esenciales entre una empresa social y una ONG es, que en el caso de la primera la obtención de ingresos es fruto de la venta de sus servicios o productos, mientras que como ya he mencionado las ONG u OSAL, dependen de la filantropía y sus donantes. Por otro, la diferencia entre las iniciativas con responsabilidad social corporativa y emprendimiento social, reside en que en el primer caso se trata de una organización basada en unos modelos de negocio sustentados en el emprendimiento convencional capitalista, que a su vez contempla unos estatutos de responsabilidad social y medioambiental como guía de acción, pero que no suponen el fin principal de su actividad económica. En el segundo caso se establece desde el inicio alrededor de un núcleo y unas estrategias cuya finalidad es la de generar un impacto responsable y positivo en la sociedad mientras desarrolla una actividad económica basada en el principio de retorno social de la inversión. Podríamos concluir que en una empresa social las relaciones laborales se basan en la equidad, los vínculos con el medioambiente se basan en una perspectiva holística de pertenencia y respeto, y por ultimo tiene la guía de acción enraizada en unos principios éticos que responden a las expectativas de la sociedad en la cual desarrolla su actividad ${ }^{4}$.

Debido a los aportes de la tercera revolución industrial, la conciencia ciudadana parece ser mayor, en algunos ámbitos. Hoy en día, tenemos más acceso a la información acerca de cómo funcionan las empresas, por lo que nuestra capacidad para tomar decisiones que repercuten en el ámbito social a través de nuestras elecciones de consumo debería ser mayor ${ }^{5}$. A este nuevo fenómeno va asociado un nuevo tipo de consumidor al que se suele denominar como "consumidor responsable" lo que habilita un nuevo ecosistema en el que el emprendimiento social es posible, ya que en términos microeconómicos pueden aportar servicios en base a una gestión más razonable de los recursos ${ }^{6}$.

Cabe decir que existen dos líneas ideológicas principales bien definidas: por un lado, podría ser concebido como una variante del emprendedor tradicional, y por otro se trataría de una categoría aparte de los emprendedores de negocio ${ }^{7}$.

Como veremos en el caso de estudio que mencionaré más adelante, nuestra orientación va dirigida en una segunda línea, ya que lo entendemos como un tipo de persona con una adquisición elevada de capital social, cuyo objetivo es el de dar solución a un problema comunitario en aquellos sectores de la economía, donde la iniciativa privada tradicional, la acción del Estado y las organizaciones sin ánimo de lucro son ineficaces, y muy importante, cuyos parámetros de éxito se sustentan en lo que denominaríamos SROI o retorno social de la inversión y no en los beneficios económicos netos.

\section{Trayectoria histórica del emprendimiento social en Japón}

Haciendo una breve revisión de la historia socioeconómica de Japón, podemos afirmar que la sociedad japonesa ha tenido una rica tradición de lo que ahora llamaríamos emprendimiento social, particularmente en el diseño

Los acrónimos mencionados en el texto, aluden en el caso del concepto de ONG a organizaciones no gubernamentales, sin ánimo de lucro, y en el caso de OSAL, a organizaciones sin ánimo de lucro. Tal y como iremos explicando más adelante, tanto el rango de acción como su constitución jurídica son distintos.

Ibata, K. (2005); Valcárcel, M. (2012).

Sería necesario observar analíticamente, si la capacidad crítica ha aumentado e ido de la mano con la apertura y democratización de la información. Bornstein, D. / Davis, S. (2012); Kaneko, I. (2013).

Martin, A. y Rivera, I (2014). 
y la utilización de soluciones culturales capaces de generar un equilibrio entre la competencia y cooperación, promoviendo con ello el desarrollo de las comunidades locales. Para ilustrarlo, podemos aludir una serie de sistemas que se fueron modificando y difuminando, con el avance de la modernidad y la transformación de las estructuras de solidaridad.

Desde el siglo VII podríamos rastrear fluctuaciones de un sistema denominado yuigumi (結組) particularmente importante en Okinawa y del que incluso quedan algunas reminiscencias hoy en día en el mundo rural, especialmente en las aldeas de estilo gassō zukkuri (合掌造り). Principalmente, hablamos de la conformación de unas dinámicas relacionales basadas en el intercambio mutuo de mano de obra y conocimientos, con el objetivo de mantener las actividades agrícolas, como la siembra y la cosecha de arroz y la recolección de los cultivos de té. La permuta de fuerza laboral fue importante también en la construcción y reparación de viviendas en pequeñas comunidades rurales y lo sigue siendo en las aldeas gassō como Mitama en Kioto, Shirakawa- Go en Gifu o Gokayama en Toyama ${ }^{8}$. Estas actividades de asistencia mutua también ejercían una labor importante en la gestión de los recursos acuáticos y madereros comunes, e igualmente en la prevención de incendios y reparación de infraestructura comunitaria tras desastres naturales. En conclusión, podríamos decir, que estaríamos ante un sistema sustentado en una asociación vecinal basada en el territorio y la horizontalidad relacional, cuyo desempeño económico competitivo-colaborativo y una racionalidad sustantiva, basada en el principio de musubi-tsunagari ( 結び・繋がり), jugaban un papel preponderante, constituyendo los ejes de la cohesión social y la prosperidad de la comunidad'.

Otro de los sistemas que se remonta a las ultimas décadas del siglo $\mathrm{XI}^{10}$ y guarda ciertas similitudes con el emprendimiento social contemporáneo, es el sistema $z a$ (座). El origen proviene de miyaza (宮崎), organizaciones o grupos de servicio en el templo. Originalmente, el $z a$, representaba el 'asiento' en las ceremonias religiosas lo que implicaba competencia por un número limitado de puestos. Este se fue transformando en una institución económica que restringió la entrada y la competencia dentro de un mercado específico de productos o servicios para obtener ganancias económicas para sus miembros (que eran libres de renunciar a él). A su vez su auge se debió a que también proporcionaba ganancias económicas a la autoridad política que le permitía asegurar esos beneficios. Se trataba de una herramienta comunitaria cuyos objetivos, aparentemente opuestos, eran los de crear un marco para la competencia basada en el mercado, por un lado, y una armonía en la comunidad, por el otro. Según las fuentes escritas provenientes del templo de Iwashimizu Hachimangū, alrededor de 1220 se confirma la existencia de alrededor de una docena de $z a$ en Kioto (para carpintería, pintura, aceite de lámpara, madera, tejado de paja, fabricación de tapetes, varios tipos de telas, etc.) patrocinados de diversas maneras por Kōfukuji, Tōdaiji, Iwashimizu Hachimangu, otros templos y santuarios, y por algunos nobles de la corte ${ }^{11}$.

El último de los sistemas que podemos encontrar a partir del siglo XIII y que guarda similitudes con las asociaciones de crédito rotativo del mundo rural europeo, es el sistema $k \bar{o}$ (講). Los campesinos formaban organizaciones excluyentes de índole religioso-social que ejercían tanto funciones de sociabilidad como crediticias. En general, se trataba de gōno (豪農) o granjeros y agricultores más o menos prósperos, que a través de la concurrencia y con una intencionalidad en muchos casos de incrementar su estatus, generaban un sistema de fondos en su comunidad a través de las ganancias obtenidas de las redes de comercio regional y nacional. Veremos una expansión importante de este sistema de la mano del famoso Ninomiya Sontoku (1787-1856) a principios del siglo XIX. Este moralista y líder agrario, en cuyo honor se construyeron santuarios póstumamente en los distritos rurales de Tokio, ejerció una labor notable creando instituciones como los Gojōkō (五常講) o “Sociedad para el Reembolso de la Virtud”"12. El sistema de planificación o presupuesto de Ninomiya, sin embargo, no fue concebido solo en términos de necesidades personales inmediatas, por el contrario, se trataba de una proyección de larga duración. La gente adscrita a estas "cooperativas agrarias" tuvo que contribuir al bienestar de los demás generando este tipo de fondos. Cada miembro de la unión de la aldea podría pedir prestados fondos sin intereses durante 100 días, y a su vez, en caso de incumplimiento, los miembros compartían el costo.

En palabras de Ninomiya Sontoku:

«Así como el individuo comparte la vida de una comunidad y se beneficia de las contribuciones de sus predecesores, este también debe contribuir al bienestar general. Dado que el bienestar de cada individuo está ligado al de la comunidad, los sufrimientos de algunos, si no se les ayuda en tiempos de angustia, eventualmente afectarán las vidas de los demás y frenarán el progreso de todos. La marca de una comunidad civilizada es lo que proporciona

En 2016 cuando me encontraba en una estancia de investigación en la Universidad de Ritsumeikan, en una visita a un pueblo llamado Mitama, hablando con los habitantes autóctonos de ésta aldea, pude confirmar que el sistema Yui seguía manteniéndose. Debido a que cada 2 o 3 décadas se suelen llevar a cabo los trabajos de fukkyuusangyou (復旧産業) para reparar los tejados y techumbre, es necesaria la implicación de la comunidad. Dado el costo que tendría la contratación de mano de obra ajena, todos los propietarios de casas minka (民家) se ayudan entre si durante las reparaciones.

Wakamori, T. (1980); Kikuchi, T. (2005); Kaneko, I. (2013).

10 El primer registro de un $z a$ perteneciente al templo Tōdaiji tiene fecha de 1097. Se trataba de un $z a$ para los cortadores de leña del bosque del templo. Yamamura, K. (1973).

12 Bajo nuestra perspectiva la traducción literal podría ser: "sociedad de las 5 virtudes cardinales confucianas", no obstante, con la intención de sintetizar en una máxima la idiosincrasia de esta entidad, considero más efectiva la traducción propuesta, debido a la función moralizante y crediticia que desempeñaba. Se trata de una traducción realizada por el autor del presente artículo. 
ayuda mutua, reconociendo la deuda que cada hombre tiene con sus semejantes y con sus antepasados por su contribución al bienestar general.»> ${ }^{13}$

En las comunidades agrarias desarrolladas en Odawara, se usó la experiencia histórica del sistema $k \bar{o}$ para expandir estas cooperativas de crédito voluntario ${ }^{14}$.

Podríamos hablar de una gran variedad de iniciativas, que se basan en los sistemas anteriormente mencionados y que guardan elementos en común con el emprendimiento social como por ejemplo la asociación Kaitokudō (懐徳) ${ }^{15}$ en Ōsaka, en 1724, los Ōmi shōnin (近 江 商人) ${ }^{16}$ de Shiga y el sistema sanpo yoshi o el sistema kamadokin (䆠金) $)^{17}$ en Kioto $^{18}$.

No obstante, hay tres hitos históricos que proyectan grandes cambios para el contexto sociopolítico y cultural a través de fuertes intentos de homogeneización, que de alguna forma fueron eliminando, ralentizando o reestructurando este tipo de iniciativas. Por un lado, la Restauración Meiji y la construcción de Japón como un Estado Nación, de corte europeo, a través de un proceso de reorganización y recentralización del poder a manos de un gran sistema de burocracia. Por otro, alrededor del final de la Gran Guerra o Primera Guerra Mundial, se inicia un proceso que generaba dinámicas sociales de barrera para las anteriores formas de organización. Cuando la economía japonesa se centró en las industrias pesadas desarrolladas de la mano de los zaibatsu (財閥) y otras grandes corporaciones, se generó una tendencia en la que el individualismo y la espontaneidad, se iban perdiendo en pos de un sistema de relaciones basado en la verticalidad y el familismo. Aquí la invención de la tradición llevada por los juristas de Meiji, en torno a la ie (家), generó un discurso político que hizo creer al japonés medio, que históricamente hubo un modelo único de familia. De facto, esta metáfora, se acabaría elevando en el siglo XX al grado de norma jurídica ${ }^{19}$.

Más adelante, con la ocupación americana tras 1945 vemos procesos de aperturismo inicial con la construcción de cooperativas de consumidores alrededor del año 1948, la aparición de nuevos movimientos sociales como Behiren (ベ平連), las protestas estudiantiles de mayo del 68, el movimiento antinuclear, el Kyodoren (法人 共同連) etc., que a su vez generaban pequeños espacios para el emprendimiento social. Aquí observamos varios flujos de interacción entre los movimientos y sus contextos, combinados con la evolución de la cultura, de la red, y las perspectivas relacionales que prefigurarán el germen de una primera generación incipiente de emprendimiento social. Esta primera generación tendría una orientación débil de mercado, una acción social regionalmente limitada y con bajas pretensiones de transcender su marco constitutivo.

No obstante, si analizamos la estructura de oportunidad política de la época y las formas de movilización de recursos, observamos que generan un contexto limitante, que acabará soterrando gran parte de estas iniciativas debido a la sinergia generada a través de la combinación que denominamos como tetsu no sankaku (鉄の三角). Hablamos del triángulo de hierro compuesto por: los burócratas del Tsūsanshō (通商産業省) o MITI, los keiretsu (系列), y los grupos políticos como el Jiyū-Minshutō (自由民主党) o PLD.

El entonces fuerte estado de desarrollo japonés impuso restricciones estructurales muy contundentes a las iniciativas sociales y dio carta blanca a un desarrollo industrial que no tenía en cuenta las consecuencias de su actividad económica, tal y como demuestran los movimientos sociales que a mediados de la década de los 60 ,

13 Ninomiya, S. (autor) / Theodore de Bary, Wm (trad.ed) (2001).

"Just as the individual shares in the life of a community and benefits from the contributions of his predecessors, so must he contribute to the general welfare. Since each individual's welfare is bound up with that of the community, the sufferings of some, if unaided in times of distress, will eventually affect the lives of others and hold back the progress of all. The mark of a civilized community is what it provides for mutual aid. In agrarian communities, this should take the form of a voluntary credit union." (pp. 373-374). [trad. castellano Mario Malo].

14 Sansom, G. (1962); Kaplan, E. (2003); Williams, Y. (2003).

15 Respecto a la Kaitokudō se trataba de una organización creada en Ōsaka en 1724 por comerciantes que promovían la educación moral y formas de mejorar la agricultura y regular el mercado.

16 Por otro lado como dice Ogura (1924), los Ōmi shōnin, fueron un grupo inicial de 100 aldeanos viajaron por todo el país vendiendo medicamentos, textiles y otros productos de su región natal de Ōmi (Shiga). Los Ōmi shōnin expandieron gradualmente sus operaciones y crearon una red nacional de tiendas, realizando negocios desde Hokkaidō hasta Kyūshū. Tuvieron que construir relaciones de confianza a largo plazo y asegurarse de que respetaran las comunidades en las que operaban bajo el sistema sanpo yoshi, a la vez que desarrollaban iniciativas de redistribución del capital en sus comunidades.

17 En 1869, los residentes de la ciudad de Kioto establecieron las bangumi shogakko (escuelas primarias comunitarias de la comunidad local autónoma) en cada uno de los 64 distritos de la ciudad. Estas escuelas fueron apoyadas por ciudadanos comunes. Se pidió a cada familia que decidiera la cantidad de donación por sí sola con un criterio aproximado, que era proporcional al número de kamado (hornos de cocina) en sus hogares. La idea era doble: una casa con más kamado debía ser más próspera y, por lo tanto, se esperaba que contribuyera más, y la otra era generar un elemento de competencia entre los ciudadanos.

18 Mitsuyoshi, H (1911); Ogura, E (1924).

19 Villaseñor, F. (2010).

La tradición nihonjinron (日本人論) o teorías sobre la peculiaridad o esencialidad insular de los japoneses han alimentado una serie de interpretaciones sobre las estructuras y mentalidades de la sociedad japonesa, hasta el punto que podemos sugerir que muchas de ellas prefiguran una mentalidad ficticia elaborada por una gran cantidad de pensadores y escritores. Por ejemplo, en 1972 la famosa antropóloga Nakane Chie (1926) en su obra Tateshakai no Ningen Kankei, habla de una estructura de jerarquía vertical y homogénea como fundamento de la sociedad japonesa que sustentará su discurso a lo largo de gran parte de su producción intelectual. Por otro lado, en 1979, Murakami Yasusuke, Kumon Shumpei y Seizaburō Satō, en su obra, Bunmei Toshite no Ie Shakai usaron el concepto ie o familia como metáfora de un principio organizativo en el que primaban colectividad y verticalidad en las relaciones sociales y cuyo origen se estaría entre los siglos VII-X. Hoy en día este sistema paternalista y patrilineal sigue reflejándose en diferentes grupos socioeconómicos, y persiste desde el ámbito familiar, a la política y el mundo empresarial japonés a través de un discurso que legitima las relaciones de poder dentro de sus entornos. Como he ido mencionando anteriormente, a pesar de que la sociedad japonesa ha tenido componentes de verticalidad y colectividad en su estructura básica, podemos ver que el individualismo y el espíritu emprendedor también han sido exhibidos en los diferentes estratos socioeconómicos a lo largo de diferentes periodos históricos. 
comenzaron a organizarse en torno a problemas ambientales en regiones muy contaminadas de Japón (enfermedad de Minamata en la prefectura de Kumamoto, de itai-itai en Toyama). Hemos de tener en cuenta que el país había pasado por la Segunda Guerra "Mundial" ${ }^{20}$ y subsecuentemente por la pobreza y la desarticulación social, por ello una gran parte de los japoneses compartían la opinión del Gobierno y de la burocracia de que el crecimiento económico era el objetivo nacional más importante. El estado del desarrollo hasta los años 90 gozó de un fuerte apoyo público para las políticas orientadas a un modelo neoliberal, por ello los problemas no económicos, políticos o cívicos, como la expansión de los derechos de los ciudadanos o el respeto al medioambiente ocuparon un segundo plano ${ }^{21}$.

\section{Segunda generación de emprendimiento social}

A principios de los años 90 con la explosión de la burbuja económica, hōkai (崩壊), se reactivaron los shimin undō (市民運動) o movimientos de base, preparando el sustrato de una segunda generación de emprendimiento social que aparece tras los sucesos acaecidos en 1995 con el gran terremoto de Kobe o Hanshin Awaji Daishinsai (阪 神・淡路大震災 $)^{22}$.

La falta de respuesta del Gobierno ante este incidente reveló efectivamente la importancia de contar con organizaciones de la sociedad civil como las NPO, asociaciones vecinales como las chōnaikai (町内会) ${ }^{23}$ e incluso iniciativas semiprivadas con las que se pueda proporcionar asistencia en caso de crisis. Tanto los funcionarios como los grupos cívicos realmente estaban muy mal preparados para responder a la crisis, y la debacle administrativa llevó al Gobierno japonés a iniciar una reforma política en 1998 para mejorar el sistema de gestión de desastres ${ }^{24}$. Las reformas políticas realizadas en 1998 se encaminaban hacia a la descentralización del poder estatal, mientras que la ley de NPO alentaba el crecimiento de la sociedad civil dotando de estatus legal a las NPO ${ }^{25}$. De hecho, la mayoría de las OSFL incorporadas aparecieron en esta época. De las 1.910 OSFL que respondieron a una encuesta realizada por el "Instituto de Investigación de Economía, Comercio e Industria" en 2003, el 82,5\% se formó después del gran terremoto de Hanshin o Kobe en enero de 1995, y el 67,5\% se incorporó después de la promulgación de la Ley de OSFL en diciembre de 1998. Estos datos dan fuertes indicios de que la ley NPO aceleró la incorporación de las OSFL en la sociedad japonesa ${ }^{26}$.

Fue alrededor de esta época cuando la segunda generación de emprendimiento social empezó a generar en varias localidades de Japón lo que conocemos como empresas comunitarias y empresas sociales.

La mayoría de las asociaciones-organizaciones que pertenecen al grupo de segunda generación, creadas en el contexto citado tienen un fuerte sentido de misión social, de hecho, el concepto de voluntariado o b/vorantia (ヴオランチア $)^{27}$ nace en esta época. Si hablamos de su orientación al mercado, tienden a ser semi-pasivas, ya que la mayor parte de los ingresos proviene de cuasi mercados y de la contratación externa por parte de los Gobiernos y salvo excepciones, suelen carecer de enfoques innovadores ${ }^{28}$

\section{Tercera generación y Tōhoku post Fukushima}

A partir del año 2000, el Estado generó un contexto de cuasi mercado basado en el seguro nacional para el cuidado de personas mayores, que para 2011 ya suponía algo más de 92 mil millones de dólares estadounidenses. Aquí se puede ver un proceso de concurrencia en el que compiten organizaciones sin fines de lucro, con empresas con fines de lucro.

En este contexto se empieza a producir un desglose dentro de las NPO (OSAL). Dependiendo del desarrollo de su actividad económica y de la finalidad de esta, cabría dividirlos en dos grandes subgrupos. Por un lado, aquellos que mantienen su dinámica constitutiva inicial y llevan a cabo labores como NPO con pocos o ningún ingreso del trabajo que realizan como "sin ánimo de lucro, puro". Por otro, aquellos que transforman su actividad primigenia (o se constituyen inicialmente bajo parámetros de emprendimiento de largo recorrido) y obtienen ingresos

\footnotetext{
20 En la línea de lo que propone Dussel no compartimos la validez de este término, ya que implica un ocultamiento de la otredad de base eurocéntrica, de aquellos que quedan fuera de la Historia. Comprendemos que se trata de un concepto que solo alude a un conflicto intraeuropeo al que se añade Japón, la URSS y Estados Unidos, por la hegemonía del mundo capitalista y que excluye las periferias y por ello una parte importante del mundo. Véase Dussel, E. (1994).

21 Garon, S. (1997); Schwartz, F/ Pharr, S. (2003); Pekkanen, R. (2006); Funabashi, H. (2006).

22 Jinno N./ Makisato T. (2012).

23 Las chōkai son grupos de base comunitaria organizados por voluntarios que tienen como objetivo lograr autogobierno y asistencia mutua. Imada, M. (2003); Sorensen, A. (2002).

25 Con este movimiento el Estado intentó conseguir deslocalizar una parte de sus servicios sociales, usando mano de obra voluntaria a bajo coste para poder paliar el déficit económico. Por las características espaciales y la finalidad del artículo es imposible desarrollar aquí esta argumentación.

26 Ogawa, A. (2010); Pradyumna, K. (2011); Broadbent, J./ Brockman, V. (2011).

27 Según el sistema de romanización Hepburn-hebon-shiki se debería de escribir con b, no obstante, la mayoría de especialistas lo escriben con $\mathrm{v}$ debido a que proviene del inglés volunteer.

28 Gill, T./ Steger, B./ Slater, D. (eds.) (2013).

En particular, los cuasi mercados constituyen un gran mercado (el más grande es el de la atención a personas mayores con un tamaño de mercado de \$ 92.4 mil millones en 2011) del cual muchas empresas sociales y sin fines de lucro de tipo emprendedor obtienen sus ingresos sostenibles. Ya que los cuasi-mercados están bajo la esfera control del Gobierno, los cambios de política adecuados y / o las operaciones flexibles de las políticas darían lugar a una ampliación sustancial de las actividades por parte de las organizaciones sociales y las organizaciones de emprendimiento.
} 
sustanciales generados tanto en un cuasi-mercado, basado en esquemas de subcontratación regional y nacional por el Gobierno, como en nuevas orientaciones al mercado en varios ámbitos que después trataremos, como el energético, el agro tecnológico o el sector del turismo. Podríamos denominar a este grupo como "sin ánimo de lucro, de tipo emprendedor" 29 .

Entenderíamos, por lo tanto, que la injerencia política en las iniciativas estatales de la primera década del siglo XXI, propició otras dinámicas de movilización de recursos, que unidas a la difícil escalabilidad que presentaban los proyectos en las OSAL de segunda generación, dieron lugar a la creación del sustrato necesario para que aparezcieran las organizaciones denominadas "sin fines de lucro de tipo emprendedor".

En este periodo, se empieza a vislumbrar una tercera generación de emprendimiento social, cuyas características difieren de las de sus predecesores en los principales ámbitos de referencia que hemos ido mencionando. Son activos en las tres dimensiones del emprendimiento social, es decir, innovación, orientación al mercado y una acción social más expansiva. Estas nuevas organizaciones tienen una progresión económica que les permite diseñar y llevar a cabo iniciativas con un mayor rango de impacto social.

Derivados de una nueva estructura de oportunidad política, fruto de la deslegitimización del Gobierno y la iniciativa privada (TEPCO y el lobby nuclear), el triple desastre de Fukushima, produce otros vectores de orientación, que supones un cambio tanto para los movimientos como para las iniciativas sociales. La acción y presión colectiva, se fue desarrollando en un marco contextual en el que el descontento generalizado se va capitalizando por parte de los movimientos de base como Shiroto no Ran (素人の乱), Hangenren (反原連), etc. Con la ayuda de las redes sociales, se consiguió movilizar diferentes fuentes solidaridad y capital social que ayudaron a crear nuevos niveles de empatía y conciencia ${ }^{30}$.

Entendemos, que por un lado los desastres suelen desencadenar, temporalmente, nuevos escalones de consciencia a través de un tipo de destrucción readaptativa y/o creativa, que reemplazan el egoísmo subjetivo por formas de solidaridad basada en la compasión. Por otro lado, la iniciativa privada suele usar estás situaciones de desastre para readaptarse y aprovechar nuevas oportunidades de mercado. Aprovechando la conmoción profunda los ciudadanos, imponen rápidamente unos planes de reforma que transfiere los activos públicos al sector privado ${ }^{31}$. Estas dos respuestas de oposición muestran las diferentes formas de poder que tenemos en caso de una fuerza extrema de la naturaleza. Uno es el poder de resistencia de los ciudadanos para vivir con tales fuerzas al hacer que sus comunidades sean más abiertas. El otro, es el poder dominante de los actores económicos más pujantes del sistema, que usan el desastre como una "hoja en blanco" en la que desarrollar su propio esquema ${ }^{32}$.

Los casos de estudio que analizaremos a partir de ahora, contemplan una vía intermedia, en la que la sociedad civil responde al desastre configurándose inicialmente, como NPO a través de diferentes pulsiones de particulares comprometidos con su comunidad, y se acaban adaptando con cierto éxito a un marco de mercado general, bajo los parámetros del emprendimiento social. Ello, ha generado dinámicas de concurrencia con fuerzas del capitalismo neoliberal y de alguna manera, en ocasiones tan solo temporalmente, ha conseguido desplazar viejas prácticas insolidarias y asociales de diferentes corporaciones que operaban en diferentes zonas de Tōhoku.

Concretamente, estamos hablando de una localidad situada en la prefectura de Iwate, llamada Tōno ${ }^{33}$. En esta, zona han proliferado diferentes grupos de emprendedores sociales, de primera, segunda y tercera generación desde 1994, como por ejemplo Ayaori Yume wo Sakaseru Josei no Kai ${ }^{34}$, Tsukimoushi Farmers Net ${ }^{35}$, NPO Tōno Eco $\mathrm{Net}^{36}$, Tōno Private Lodging Association ${ }^{37}$, etc., que cuentan con un fuerte arraigo y compromiso territorial y que generalmente han basado sus actividades en el turismo verde, la agricultura sostenible y en general actividades que tienen la premisa de mantener cierto equilibrio con los nichos ecológicos que habitan. En 2003 pasaron a formar parte de una NPO llamada Yamasatonetto, y resultarían fundamentales en la gestión del voluntariado post-

Tanimoto, K. (2008); Sugimoto, Y. (2010).

David, P./ Bill, E./ García, S. (2011).

Tal y como se puede comprobar si se analizan las dinámicas de actuación y las responsabilidades asumidas por TEPCO y el propio Gobierno.

Brannigan, M. (2015); Mullins, M./ Nakano, K. (2016); Tamura, A. (2018).

Famosa por ser cuna del excelso antropólogo considerado padre de los Minzogaku (民俗学), Yanagita Kunio (1875-1962), el cual, justamente aquí, como refleja en su obra Tōno Monogatari (東野物語), redescubrió una parte importante del folclore japonés. También fue el lugar de inspiración para muchas de las novelas de Miyazawa Kenji (1896-1933) originario de Hanamaki, una pequeña ciudad muy cerca de Tono.

34 “Asociación de mujeres de Ayaori dispuestas a realizar sus sueños". Se trata de una asociación de mujeres cuya principal actividad hoy en día se organiza alrededor de la economía circular alrededor del sector agropecuario. Llevan operando desde 1994 en Tōno, sus actividades incluyen la gestión de un restaurante de granja llamado "Yumesaki Chaya", en el que cocinan sus propios cultivos y así como unos baños naturales en el entorno de los arrozales. Por otro lado, en la última década han desarrollado nuevos tratamientos de basura utilizando EM (microorganismos efectivos). Estaríamos ante un grupo de emprendimiento social que ha ido transformándose y mezcla componentes de las 3 generaciones de emprendimiento, tienen un impacto eminentemente local, pero son innovadores como demuestran los premios: 2001 Agricultura, Silvicultura y Pesca en la Alimentación, Desarrollo Comunitario para Mujeres 2002 e Innovación y Actividades de Mayores en Zonas Rurales en 2014.

35 Tsukimoushi Farmers Net es grupo de agricultores lanzó sus propias actividades para promover el turismo verde.

36 NPO Tōno Eco Net, fue fundada con el deseo de restaurar el paisaje natural de colinas y campos en Tōno para que pueda transmitirse a las generaciones posteriores. Bajo iniciativas de ecoturismo intentan contribuir con la comunidad mientras preservan la naturaleza, trabajan para desarrollar el medio actividades de conservación en estrecha colaboración con la comunidad local y la administración. También identifican y utilizan una amplia gama de productos regionales.

37 Tōno Private Lodging Association ayuda a los huéspedes a encontrar no solo alojamiento privado si no también actividades de "experiencia". Hay 135 miembros registrados desde marzo de 2014, que trabajan para promover viajes educativos, actividades de experiencias agrícolas e intercambios por iniciativa propia de los ciudadanos. 
desastre $^{38}$. Por ello, nos centraremos en los desarrollos tras el 11 de marzo de 2011, y en las sinergias entre los diferentes grupos.

Debido a la especial ubicación geoestratégica de Tōno (está a menos de 1 hora de muchos de los lugares más afectados por el tsunami), se organizaron desde el primer momento, el gobierno prefectural, el ejército, las NPO, el jichikai (自治体) y los grupos de voluntarios, para ofrecer soporte logístico a las comunidades afectadas por el desastre, como Kamaishi, Otsuchicho o Rikuzentakata. A finales de marzo de 2011, la NPO conocida como Magokoro Network junto a la concejalía de salud, tuvieron la capacidad de aglutinar a varios colectivos de la sociedad civil como la NPO Yamasato Kurashi Network, compuesta por los grupos que he citado anteriormente, y voluntarios individuales, para dar respuesta al incidente y trabajar en la recuperación de las zonas damnificadas.

Con estos grupos de voluntarios había una mujer de origen italiano, llamada Renata Piazza, que sirve de prototipo para entender que es un social entrepeneur y un transition designer de tercera generación y como en su conjunto este tipo de figuras ejercen un papel de vertebradores territoriales. Renata Piazza, después de haber desarrollado un recorrido profesional en el ámbito privado y público japonés en varias entidades como bancos, aerolíneas, Casa Asia, etc., a través de su experiencia en el voluntariado en Fukushima y de su conexión con la NPO Magokoronetto y la NPO Yamasatonetto ${ }^{39}$ decide en 2013 desarrollar un proyecto de emprendimiento social en Barcelona y en 2016 se traslada permanentemente a Tōno. Dicha iniciativa recibe el nombre de Hasekura 2.040. cuyo objetivo primordial, es el de ayudar a la recuperación económica, a la dinamización de algunas zonas afectadas por el desastre. Como explicaremos en los siguientes párrafos, existen varios objetivos principales, entre los que se encuentran el de transition designer counseling, para ayudar con la transición hacia un sistema de producción y manufactura ecológico y responsable socialmente que vertebre el territorio. Por otro lado, se promueve intercambio empresarial para PYMES empresas que contemplen las premisas SROI ${ }^{41}$ y emprendedores sociales, instituciones y estudiantes de posgrado de Japón y España. Se intenta con ello crear nuevas relaciones interpersonales entre emprendedores sociales de ambos países y potenciar el intercambio de ideas y la internacionalización. Entre sus prioridades están pequeñas empresas japonesas con un papel activo en la reconstrucción de Tōhoku y pequeñas empresas españolas que se hayan visto afectadas por la crisis.

Para ilustrar y entender el contexto demográfico en el que aterriza este proyecto, sería necesario observar la siguiente tabla ${ }^{42}$.

\begin{tabular}{|l|l|l|l|}
\hline & TŌHOKU & JAPÓN & TOKIO \\
\hline Área Total & $66.889 .55 \mathrm{Km}^{2}$ & $337.944 \mathrm{Km}^{2}$ & $2.187 .66 \mathrm{Km}^{2}$ \\
\hline Población & $9.335 .636(2010)$ & $128.057 .352(2010)$ & $13.167 .876(2010)$ \\
& $9.020 .531(2015)$ & $127.160 .103(2015)$ & $13.493 .245(2015)$ \\
& $8.682 .336(2019)$ & $126.310 .000(2019)$ & $13.929 .286(2019)$ \\
\hline Más de 65 años & $28 \%$ y 37\% alrededor de la costa. & $24 \%$ & $19 \%$ \\
\hline Densidad poblacional & $136 / \mathrm{Km}^{2}$ & $338 \mathrm{Km}^{2}$ & $6.146 \mathrm{Km}^{2}$ \\
\hline Renta per cápita (2018) & $33.345 \mathrm{US} \$$ & $40.849 \mathrm{US} \$$ & $66.236 \mathrm{US} \$$
\end{tabular}

Observamos en ella como la problemática del envejecimiento demográfico y la pérdida de población son especialmente preocupantes en la región de Tōhoku sobre todo después del triple desastre. Vemos como de 2010 a 2018, se está produciendo un descenso demográfico generalizado, en Japón y por ende en Tōhoku. Por otro lado, no obstante, también vemos como se produce un trasvase migratorio desde la periferia hacia Tokio.

Por otro lado, es importante mencionar que el envejecimiento poblacional en Tōhoku es especialmente preocupante, sobre todo en la costa. A pesar de estas dinámicas poblacionales, todos esos nuevos espacios de empoderamiento y de reinvención abiertos por el 11 de marzo de 2011, hacen que una pequeña parte de los jóvenes que habían emigrado principalmente al área de Kantō y Kansai, especialmente, a las ciudades Tokio y Osaka por ser zonas con mayores posibilidades de empleo, volvieran a la zona de Tōno, Hanamaki, Rikuzentakata, Kamaishi, Yamamoto y otros pequeños pueblos y ciudades de Iwate y Miyagi.

\footnotetext{
8 Son encuestas y entrevistas realizadas por mí, a los responsables de cada entidad, a través de un cuestionario semiestructurado, durante el mes de febrero 2018 y telemáticamente durante mayo y abril de 2019.

39 Hablamos de varios proyectos, que mezclan, por un lado, iniciativas grupales sin ánimo de lucro que acaban transformándose en formas de emprendimiento social de segunda y tercera generación.

40 El propio nombre alude a Hasekura Rokuemon Tsunenaga, uno de los artífices de la misión diplomática Keichō, entre cuyos objetivos se encontraba el de crear relaciones comerciales con Felipe III en el siglo XVII, y 2.0 hace referencia al intento de crear nuevos lazos transnacionales e internacionales entre Japón y España en un inicio y también con Sicilia en la actualidad.

41 Los acrónimos aluden a pequeñas y medianas empresas y al retorno social de la inversión.

42 Elaboración propia a través de: www.metro.tokyo.jp; www.meti.go.jp; www.stat.go.jp (2019).
} 


\section{Tipologías migratorias y población autóctona.}

Podríamos establecer cuatro tipologías grupales para las zonas en las que se desarrollan estas iniciativas. Dentro de estas se encuentra el grupo que he mencionado antes, denominado U-turn y J-turn ${ }^{43}$. Son aquellos que han vuelto desde las grandes urbes a sus lugares de procedencia o a zonas rurales desde su provincia de origen. Estos abanderan nuevos proyectos creando principalmente start ups agro-tecnológicas e iniciativas de turismo verde, relacionadas con la revalorización del patrimonio natural y cultural y cuyas actividades van dando forma a los nuevos contextos de machizukuri (町作い). En general, hablamos de individuos de generaciones nacidas en los años 80 durante la burbuja económica, y de cuyos testimonios se extrae la conclusión, de que no están dispuestos a pagar el coste de social que implica vivir dentro del tipo de dinámicas económicas de una ciudad como Tokio u Osaka. Hablamos del karōshi ( 過労死), suicidios, fenómenos de ruptura social como los hikikomori (引きこもり), de precariedad laboral y del hacinamiento que representa vivir en urbes de grandes magnitudes.

Hay también un componente relacional e identitario, en el que la tendencia al desarraigo en un contexto anómico genera problemas notorios. Podemos ver que, entre otras cuestiones, debido a las relaciones líquidas y la tendencia al simulacro, que se dan en este tipo de contextos, se generan crisis identitarias de adscripción, que, en estos casos, han sido resaltadas a través de un suceso tan traumático como el triple desastre. Ello hace que en cierto modo esa idealizada idea de furusatō (故郷), del retorno al lugar de nacimiento, al origen, impulse a estos jóvenes a volver y a desarrollar un nuevo modelo socioeconómico en sus lugares de procedencia ${ }^{44}$.

Por otro lado, estarían los $I$-turn ${ }^{45}$, a los que en este caso denominamos como agentes internacionales, y que son aquellos que tenían un contacto intermitente con Japón, pero que estaba asentados en otros países y que deciden cambiar de vida y quedarse en Tōno e integrarse en la comunidad, de los que Renata forma parte.

Por último, los habitantes autóctonos de generaciones nacidas en torno a los años 60 que generalmente se dedican a la agricultura, la artesanía y el turismo. Este grupo, ante la despoblación tan abrumadora y la falta de alternativas de negocio en la zona, establecen sinergias con los dos grupos anteriores a la hora de readaptar sus negocios.

Es con estos cuatro grupos, con los que se desarrolla el proyecto Hasekura 2.0. Por un lado, Renata y los I-turn, J-turn y U-turns, capitalizando la proyección internacional de estas iniciativas, y por otro las NPO e iniciativas preexistentes anteriores a 2011, como Yamasatonetto y todas las kumiai (組合) y NPO que la integran, y que junto a la población local, trabajan reinterpretando las formas de producción.

\section{Proyectos y orientación de las iniciativas}

A pesar de que hay varios objetivos prioritarios, por cuestiones espaciales inherentes al propio artículo, tan solo hablaremos de dos. En primer lugar; deslocalizar el turismo tradicional focalizado en Tokio, Kioto y Osaka, a través de la promoción del patrimonio cultural, gastronómico y natural de la zona, intentando desarrollar un turismo sostenible responsable. Tōno Private Lodging Association, Tōno Green Tourism Study Group, Satoyama Club Yakamashi Village y Miyamori Tourism Council, son asociaciones que cuentan sobre todo con emprendedores de primera y segunda generación, pero que después de 2011 empezaron a intentar transcender el ámbito regional con sus iniciativas. Iniciativas relacionadas con sus más de treinta grupos de danzas tradicionales, como los shishi odori (獅子踊り), shika odori (鹿踊り) etc., la recuperación de la tradición oral y del folclore, y la reorientación de una gastronomía basada en sus recursos paisajísticos y naturales.

El otro objetivo es el de recuperar y revalorizar las formas de producción artesanal de alimentos y todo tipo de manufacturas. De alguna forma, siguiendo algunos patrones, que nos recuerdan, salvando las diferencias, a la labor llevada a cabo por Ernest Fenollosa a fínales del Siglo XIX y principios del XX. El trabajo etnológico realizado en 2018 con algunos habitantes del pueblo ${ }^{46}$ nos llevó a la conclusión, de que, debido a ciertas dinámicas de colonización del ser, que parten desde los centros a la periferia, vemos que todavía perdura ese estigma de vivir en el inaka (田 舎) o campo (cabría analizar la expresión inakakusai [田舎臭い] o apestas a campo), que está muy interiorizado en una parte importante de los habitantes autóctonos de estas zonas. Por ello, al inicio, los social entrepreneur siempre intentan crear conciencia en los habitantes, sobre el valor que tanto Tōno como los pueblos colindantes tienen. Esta labor, se realiza a través los transition designer o agentes del cambio mediante diferentes workshops y posteriormente, se van plantando en semilleros e incubadoras de ideas los proyectos de innovación junto a la población local.

Respecto al segundo de los objetivos, podríamos ilustrarlo a través de la explicación de algunas de las iniciativas que se desarrollan en este contexto. En primer lugar, uno de los proyectos que consideramos, que más valor simbólico

\footnotetext{
43 En el caso del concepto de U-turn se usa en el campo de los estudios migratorios para referirse a personas que se ha marchado de su lugar de origen para formarse y trabajar en una gran ciudad y que finalmente vuelven a sus lugares de nacimiento para generar un proyecto de vida. El caso de los J-Turn es similar y se diferencian en que vuelven a otras áreas más pequeñas, situadas en otras provincias que no son sus lugares de origen.

44 Durkheim, E. (1985); Braudillard, J. (1978); Zygmunt, B. (2011).

45 Originalmente este concepto se utiliza en el campo de las migraciones para referirse a un migrante que va desde una gran ciudad a una zona rural para realizar un proyecto de vida.

46 Información facilitada por miembros de las NPO Magokoronetto y Yamasatonetto, mediante varias entrevistas presenciales en su sede en febrero de 2018 .
} 
tiene es Tōno Brewing Company ${ }^{47}$. Se trata de la creación de una destilería en 2018 para hacer cerveza local artesanal ecológica, que surge del proyecto Next Commons Lab en 2016-2017 en Tōno. Resulta transcendente, debido a que hasta hace pocos años había un monopolio de casi el 100\% sobre la producción del lúpulo por parte de la empresa Kirin. Esto suponía que un porcentaje elevado de agricultores tenían que pasar a formar parte del ciclo cerrado que establecía Kirin, es decir; comprarles las semillas, los fertilizantes y venderles el 100\% de la producción. En las entrevistas realizadas a estos agricultores, mencionaron que al final las políticas de precios de la empresa les dejaban tan poco margen de beneficio que intentaron buscar alternativas en las cooperativas, pero debido a la injerencia de capital privado en estas entidades tampoco resultaba factible. Aquí entra en juego un grupo de $3 J$-turn, Hakamada Daisuke, Ohta Mutsumi y Junichichi Tamura. Originarios de Aomori, Osaka y Wakayama, respectivamente, en 20112012 realizaron labores de voluntariado en la zona de Iwate y Miyagi, ayudando a sus familiares y amigos tras el tsunami. Viendo la problemática que afrontaban conocidos y familiares en la zona de Tōno deciden trasladarse desde sus residencias en Kantō y Kansai para iniciar su proyecto, y aplicar sus conocimientos técnicos en la destilación y producción de lúpulo en colaboración con los agricultores de la zona. Ellos llevan a cabo el marketing y el branding del producto y elaboran una marca de cerveza a través de la materia prima certificada que les aportan los agricultores del lugar. Ahora venden su producto por todo Sendai y en 2018 abrieron un pub/restaurante en Tōno.

Por otro lado, observamos un ejemplo similar de colaboración, nuevos enfoques y expansión de prácticas económicas semisocietarias fruto del emprendimiento social en el área de Miyagi, en la localidad de Yamamoto. Tradicionalmente, aquí cultivaban fresas usando formas de producción preindustriales. Con el vaciamiento del mundo rural en pos de oportunidades laborales en las ciudades, los agricultores ya no encontraban relevo generacional, por lo que se estaban dejando varias explotaciones agrícolas sin usar o se arrendaban a grandes empresas que homogeneizaban los cultivos. Es aquí donde entra Hiroki Daiki, un U-turn, empresario del sector tecnológico, que después del tsunami también volvió para ayudar a sus abuelos. En 2012, junto con NPO GRA, Ichigo Inc y GRA India, usando el conocimiento familiar y su formación tecnológica, empezó a desarrollar un proyecto de economía circular. Tomando las técnicas de cultivo de sus familiares, y readaptándolas creó invernaderos altamente tecnologizados, desarrollando así el proyecto Migaki Ichigo para la producción de fresas mediante hidroponía. Algunas de las premisas de este proyecto son las de dar trabajo a otros jóvenes U-turn que han regresado a su pequeña ciudad y promover la dinamización regional en colaboración con agricultores locales, lo que se producen sinergias intra e intergeneracionales muy interesantes. Su proyecto ha tenido bastante éxito ya que en 2019 contaba con una capitalización de 34,200,000 JPY. En la actualidad vende todo tipo de productos derivados de las fresas tanto en las principales tiendas gourmet y de cosmética en Tokio como en países como Estados Unidos e India ${ }^{48}$.

Por último, un proyecto de manos de una pareja de I-turn, el Michael's Café American-Black Ship Coffee. Creado por una pareja Michael Dougan y Nitta Chizuko, los cuales tenían un contacto intermitente con Japón, pero que estaba asentados en Seattle (USA) y que deciden cambiar de vida en 2017 y quedarse en Tōno y formar parte de la comunidad. Debido a que Michael fue uno de los primeros baristas de Starbucks en Seattle, poseía un gran conocimiento sobre el tema, por lo que montó un negocio de importación y manufactura de café. Además de elaborar su propio café para el bar que montó en 2018, fundó un club de tostadores de café. En su café junto a los miembros de Hasekura 2.0 y Yamasatonetto, han iniciado proyectos de intercambio intercultural, e iniciativas para el aprendizaje de inglés de negocios, exportar los productos de Tōno a Europa y Estados Unidos ${ }^{49}$.

Hay docenas de proyectos más como los proyectos de Handcraft Tōno en los que se manufacturan artesanalmente artículos de seda producida de forma tradicional, los proyectos de doburoku y fermentación de arroz para el nihonshu, etc $^{50}$., pero lamentablemente no podemos profundizar en ellos aquí.

\section{Conclusión}

En este breve texto, hemos intentado sintetizar algunos de los mecanismos comunitarios y sistemas de cooperacióncompetitividad que se han ido dando en el archipiélago japonés, desde hace varios siglos hasta el tiempo presente. Con los breves resúmenes, de estudios de caso aquí explicados, hemos pretendido dar a conocer, someramente, cómo han funcionado las diferentes sinergias establecidas entre distintas generaciones de emprendedores sociales y de que formas se aproximan a las dinámicas del capitalismo verde o generan sus propios marcos de acción y pervivencia.

Parece ser que son procesos que están arraigando fuertemente, sobre todo en todos esos espacios reabiertos a la sociedad civil tras el desastre de 2011. Usando una idea recurrente en las obras de Deleuze y Guattari (1972-1980), en "la época de la precariedad" de alguna forma entendemos que estos procesos son posibles y están unidos a nichos de consumo con estadios de consciencia más elevados, cuya expansión permite desarrollar proyectos con una mayor

\footnotetext{
47 La información sobre Tōno Brewing Company, ha sido obtenida a través de una entrevista presencial en febrero de 2018 y de entrevistas telemáticas en abril de 2019.

48 Información por facilitada por Hiroki Daiki a través de entrevista presencial en febrero 2018 y telemática en mayo de 2019.

49 La información sobre Black Ship Coffee, ha sido obtenida a través de una entrevista presencial en febrero de 2018 y de entrevistas telemáticas en abril de 2019

50 Deleuze y Guattari (1985).
} 
carga social, en las que la mera ganancia económica pasa a un plano de mantenimiento o pervivencia, en vez de seguir orientada al crecimiento per se.

Si se mantiene en el tiempo, el emprendimiento social basado en los principios de retorno social de las ganancias, puede ser el motor de desarrollo de un nuevo modelo socioeconómico en estas zonas, que a su vez ponga en valor el patrimonio olvidado, que fije población y que permita una interacción entre diferentes generaciones con el objetivo de crear comunidades más ricas y sólidas.

\section{Bibliografía}

Bornstein, David/ Davis, Susan (2012): Social Entrepreneurship: What Everyone Needs to Know. Oxford: Oxford University Press.

Brannigan, Michael (2015): Japan's March 2011 Disaster and Moral Grit: Our Inescapable In between. London: Lexington Books.

Braudillard, Jean (1978): Cultura y Simulacro. Barcelona: Editorial Kairos.

Broadbent, Jeffrey/ Brockman, Vicky (2011): East Asian Social Movements: Power, Protest and Change in a Dynamic Region. Minnesota: University of Minnesota Press.

Deleuze y Guattari (1985): El Anti-Edipo: Capitalismo y Esquizofrenia, Barcelona, Paidós Ibérica.

Dussel, E (1994): 1492: el encubrimiento del otro: hacia el origen del mito de la modernidad, Quito, Ediciones AbyaYala.

Durkheim, Emile (1985): Las reglas del método sociológico. Madrid: Akal.

Funabashi, Harutoshi (2006): "Minamata Disease and Environmental Governance". En: International Journal of Japanese Sociology, No. 15, Yokohama, pp .7-25.

Garon, Sheldon (1997): Molding Japanese Minds: The State in Everyday Life. Princeton: Princeton University Press.

Gill, Tom/ Steger, Brigitte/Slater, Davis (eds.) (2013): Japan Copes with Calamity: Ethnographies of the Earthquake, Tsunami and Nuclear Disasters of March 2011. Bern: Peter Lang AG.

Ibata, Kathryn (2005): Innovation and Entrepreneurship in Japan: Politics, Organizations, and High Technology Firms. Cambridge: Cambridge University Press.

Imada, Makoto (2003): The voluntary response to the Hanshin Awaji Earthquake. London: Routledge.

Jinno Nahohiko/ Makisato Tsuneji (2012): Shakai kigyo nyumon: Shakai o kaeru to iu shigoto. Tōkyō: Mineruvashobo.

Kaneko, Ikuyo (2013): "Social Entrepreneurship in Japan: A Historical Perspective on Current Trends". En: Journal of Social Entrepreneurship, Taylor and Francis, Vol. 4, UK, pp. 256-276.

Kaplan, Edward (2003): The Cultures of East Asia: Political-Material Aspects. Washington: Washington University Press.

Kikuchi, Toshiaki (2005): "Hakuumatake no momotose”. En: Yamatokeikokusha, No. 24, Tokio, pp. 80-81.

Kogane, Yoshihiro (2013): Sangyo shakai no kako to mirai, Tokio, Tokai daigaku shuppankai.

Martin, Aramís/ Rivera, Igor (2014) "Revisión teórica y propuesta de estudio sobre el emprendimiento social y la innovación tecnológica”. En: Acta Universitaria, 24 (E-1), México, pp. 48-58.

Doug, McAdam (1996): Comparative Perspectives on Social Movements: Political Opportunities, Mobilizing Stractures, and Cultural Framings: Cambridge: Cambridge University Press.

Mitsuyoshi, Hirase (1911): Ōmi Shōnin. Berkeley: University of California Press.

Mullins, Mark/ Nakano Kōichi (2016): Disasters and Social Crisis in Contemporary Japan: Political, Religious, and Sociocultural Responses. London: Palgrave MacMillan.

Ogawa, Akihiro (2010): The Failure of Civil Society? The Third Sector and the State in Contemporary Japan. New York: State University of New York Press.

Ogura, Eiichirō (1924): Ōmi Shōnin no kaihatsuryoku: Kanri shisutemu to Hokkaidō kaihatsu ni miru kindai keiei. Tokyo: Chūō Keizaisha.

Pekkanen, Robert (2006): Japan's Dual Civil Society: Members Without Advocates. Palo Alto (California): Stanford University Press.

Pilling, David/ Emmott, Bill/ García, Susan (2011): Tsunami: Japan's Post-Fukushima Future. Washington: Foreign Policy Magazine.

Pradyumna, Karan (2011): Japan in the 21st Century: Environment, Economy, and Society. Kentucky: The University Press of Kentucky.

Sansom, George (1962): Japan: A Short Cultural History. New York: Appleton-Century Crofts.

Schwartz, Frank/ Pharr, Susan (2003): The State of Civil Society in Japan. Cambridge: Cambridge University Press.

Sorensen, André (2002): The Making of Urban Japan: Cities and Planning from Edo to the Twenty First. London: Routledge.

Sugimoto, Yoshio (2010): An Introduction to Japanese Society. Cambridge: Cambridge University Press.

Tamura, Azumi (2018): Post-Fukushima Activism: Politics and Knowledge in the Age of Precarity. London: Routledge.

Tanimoto, Kanji (2008): The Emergence of Social Entrepreneurship and its Strategy in Japan. Belfast: Queen's University Belfast Press.

Theodore de Bary William et al. (ed.) (2001): Sources of Japanese Tradition, Vol. 2, New York, Columbia University Press, pp. 373-374. 
Valcárcel, Mercedes (2012): “Los fondos europeos de emprendimiento social: su aplicación en España". En: CIRIECEspaña, Revista de Economía Pública, Social y Cooperativa, No. 75, Madrid, pp. 106-127.

Villaseñor, Fernando (2010): "La importancia del modelo occidental de derecho en la creación del Estado Japonés". En: Boletín Mexicano de Derecho Comparado, No. 127, México, pp. 97-126.

Wakamori, Taro (1980): "Wakamori tarō chosaku-shū" zen 16-kan kōbun dō, Shinbunsha, Tokio, pp. 83-84.

Williams, Yoko (2003): Tsumi - Offence and Retribution in Early Japan. London: Routledge.

Yamamura, Kozo (1973): The Development of Za in Medieval Japan. Washington: Washington University Press.

Zygmunt, Bauman (2011): Culture in a Liquid Modern World. Cambridge (UK): Polity.

www.metro.tokyo.jp . Tokyo Metropolitan Government. http://www.metro.tokyo.jp/index.html

www.meti.go.jp. Ministry of Economy, Trade and Industry. https://www.meti.go.jp

www.stat.go.jp (2019): 総務省「第68回 日本統計年鑑 平成31年」El 68 Anuario Estadístico de Japón 2019. Statistic

Government Bureau of Japan. http://www.stat.go.jp/naruhodo/c1data/04_03_stt.html 